\title{
Pratiques
}

Linguistique, littérature, didactique

$141-142 \mid 2009$

La synonymie

\section{Enseignement du lexique et production écrite. Un travail sur les verbes de déplacement à la fin de l'école primaire}

\author{
Claudine Garcia-Debanc, Karine Duvignau, Claire Dutrait et Michel
} Gangneux

\section{(2) OpenEdition}

Journals

Édition électronique

URL : https://journals.openedition.org/pratiques/1361

DOI : 10.4000/pratiques. 1361

ISSN : 2425-2042

Éditeur

Centre de recherche sur les médiations (CREM)

Édition imprimée

Date de publication : 15 juin 2009

Pagination : 208-232

Référence électronique

Claudine Garcia-Debanc, Karine Duvignau, Claire Dutrait et Michel Gangneux, « Enseignement du lexique et production écrite. Un travail sur les verbes de déplacement à la fin de l'école primaire », Pratiques [En ligne], 141-142 | 2009, mis en ligne le 20 juin 2014, consulté le 10 mars 2023. URL: http://journals.openedition.org/pratiques/1361 ; DOI : https://doi.org/10.4000/pratiques.1361 


\section{Enseignement du lexique et production écrite. Un travail sur les verbes de déplacement à la fin de l'école primaire}

\section{Claudine Garcia-Debanc, Karine Duvignau}

CLEE-ERSS, UMR 5263, Université Toulouse2 Le Mirail \& GRIDIFE, ERT 64, IUFM Midi-Pyrénées-Ecole Interne Université Toulouse2-Le Mirail Claire Dutrait, Michel Gangneux IUFM Midi-Pyrénées-Ecole Interne Université Toulouse2-Le Mirail

«Les mots nous intimident. [...] Souvent, nous disons : «Je ne trouve pas les mots ». [...] Pour les apprivoiser, il faut les soupeser, les regarder, apprendre leurs histoires, et puis jouer avec eux, sourire avec eux. Les approcher pour mieux les savourer, les saluer, et toujours un peu en retrait, se dire : «Je l' ai sur le bout de la langue - le goût du mot qui ne me manque déjà plus »». C'est ainsi que l'écrivain Philippe Delerm présente, dans un texte introductif, la collection qu'il dirige aux éditions Points-Seuil, qui s'intitule « Le goût des mots ». Dans l'un des ouvrages de cette collection plus particulièrement consacré aux questions de synonymie, intitulé Un bouquin n'est pas un livre, Rémi Bertrand commence de la façon suivante l'article qu'il consacre aux verbes offrir, donner, octroyer, accorder : «ces verbes désignent l'action de transmettre une chose à quelqu'un; mais chacun exprime une nuance dans le comment et le pourquoi de cette action. Synonymes par le résultat du geste ; différents par la nature du geste même. » Goûter la saveur des mots pour les apprivoiser et les choisir à bon escient lorsqu'on écrit, tel est le programme de travail des écrivains mais aussi, d'une certaine manière, celui que peut s'assigner une didactique de l'écriture.

La présente contribution ${ }^{(1)}$ s'inscrit dans la perspective d'un enseignement de la langue au service de l'écriture et de la construction d'aides lexicales à la production

(1) La conception des séances de travail qui sont présentées ici a été réalisée dans le cadre de la recherche collaborative «Enseigner le verbe : dimensions sémantique, morphologique et syntaxique ", financée par 1'IUFM Midi-Pyrénées, Ecole interne de l'université Toulouse2-Le Mirail, dans le cadre de 1'ERT 64, GRIDIFE, Groupe de Recherche sur les Interactions Didactiques et la Formation des Enseignants. Je remercie particulièrement Philippe Hitier qui a mis en œuvre ce travail avec ses élèves de CM2 de 1'Ecole Annexe Bénezet à Toulouse, Michel Gangneux et Claire Dutrait, mes collègues formateurs à 1'IUFM MidiPyrénées, avec qui a été imaginée cette démarche, Karine Duvignau et Bruno Gaume, chercheurs au laboratoire CLEE-ERSS, qui nous ont aidés à sélectionner les verbes grâce à leur logiciel PROX. 
écrite. Des travaux en didactique des langues (Noyau 2003, 2005, Kihlstedt, 2005 ) ont en effet montré que les élèves ou les adultes apprenant une langue seconde utilisent préférentiellement des verbes génériques dans des tâches de reformulation de récits. Il en est de même lorsqu' eux-mêmes rédigent des textes. L'enseignement se doit de les aider à utiliser de façon pertinente des verbes spécifiques. Le travail porte moins sur la recherche de synonymes que de co-hyponymes de verbes souvent qualifiés de « passe-partout», tels que faire, dire, mettre, aller. Nous essaierons de montrer ici, à travers l'exemple d'un travail conduit en langue sur les verbes de déplacement dans une classe de CM2, en relation avec un projet d'écriture littéraire, comment peuvent s'articuler activités de production écrite, analyse des emplois du lexique d'un domaine donné en discours et travail systématique en langue et quels effets peut avoir une telle étude sur les productions écrites des élèves.

Cet article s'efforce ainsi de répondre à une double visée, d'ordre praxéologique et d'ordre épistémologique. D'un point de vue praxéologique, il se propose de fournir aux enseignants et aux formateurs d'enseignants un exemple de mise en œuvre d'un enseignement lexical au service de la production écrite. D'un point de vue épistémologique, il prétend montrer les articulations dynamiques entre didactique $\mathrm{du}$ français et recherches linguistiques et contribuer ainsi, dans le prolongement du numéro 137-138 de Pratiques, à clarifier la spécificité de la didactique du français par rapport à l'une de ses disciplines de référence privilégiées, la linguistique.

Les enseignants se posent de nombreuses questions sur la mise en œuvre des prescriptions des programmes 2008 de l'école primaire ${ }^{(2)}$. A quelles conditions les élèves accroissent-ils leur vocabulaire passif et actif? Sur quels contenus faire porter l'enseignement du lexique ? Comment aider les élèves à utiliser un vocabulaire plus spécifique dans leurs productions écrites? Quelle place réserver à l'observation des emplois de mots dans des textes, notamment littéraires, et à un travail systématique en langue ? Quels dictionnaires utiliser en classe avec les élèves mais aussi pour ses propres préparations ? Quelle place leur réserver? La contribution présente s'efforce d'apporter quelques réponses à certaines de ces questions.

D'un point de vue épistémologique, si la didactique du français langue première peut être définie comme « discipline scientifique ayant pour objet l'étude des interventions d'enseignement permettant l'appropriation par des élèves, au sein de l'institution scolaire, dans des classes ordinaires aménagées selon des dispositifs adéquats, de pratiques culturelles, de compétences et de notions se rapportant à la pratique et à l'analyse de la langue et des discours » (Garcia-Debanc, 2008), en vue d'une amélioration des performances langagières, particulièrement des élèves de milieux socio-culturels défavorisés, on peut essayer de cerner les exigences méthodologiques qu'elle doit satisfaire, en tant que discipline scientifique. Le champ de la didactique du français est plus large que le domaine balisé

(2) Les programmes 2008 pour l'école primaire, de même que ceux de 2007 ou ceux du collège accordent une place importante à un enseignement du lexique organisé et spécifique, dont ils indiquent les enjeux : « le but de l'enseignement du vocabulaire est d'accroître le lexique des élèves (découverte et mémorisation de mots nouveaux et de leur sens) et de favoriser par l'exercice la procédure naturelle par laquelle les mots connus et compris (vocabulaire passif) se transforment en mots utilisés (vocabulaire actif) »(BO 17 avril 2007, p. 116). Ils définissent à grands traits les orientations de cet enseignement : « chaque jour, dans les divers domaines d'activité, et grâce aux histoires que l'enseignant raconte et lit, les enfants entendent des mots nouveaux mais cette simple exposition ne suffit pas pour qu'ils les mémorisent. L'acquisition du vocabulaire exige des séquences spécifiques, des activités régulières de classification, de mémorisation de mots, de réutilisation du vocabulaire acquis, d'interprétation de termes inconnus à partir de leur contexte » (p. 12). 
dans cette définition, dans la mesure où les recherches didactiques peuvent aussi décrire les pratiques d'enseignement « ordinaires » ou proposer une étude historique et épistémologique des matériels d'enseignement, des discours sur l'enseignement ou des pratiques d'enseignement. Si l'on retient la dimension d'intervention dans le champ social mise en avant dans la définition ci-dessus, les thématiques de travail de la didactique du français sont définies à la convergence de trois paramètres à prendre en compte de façon contrôlée :

- 1'existence d'une question d'enseignement, formulée par les acteurs du champ scolaire, qu'ils soient enseignants ou formateurs d'enseignants, les décideurs ou les chercheurs,

- la disponibilité de travaux linguistiques relatifs au contenu enseigné, qui peuvent fournir des cadres d'analyse et contribuer à l'élaboration de contenus d'enseignement,

- 1'existence de travaux psycholinguistiques ou sociolinguistiques permettant de baliser les conditions d'un apprentissage de la notion par les élèves.

Ces différents paramètres autorisent la formulation d'hypothèses pour une recherche didactique. Celles-ci doivent également prendre en compte les contraintes institutionnelles des terrains d'enseignement et la culture disciplinaire des acteurs de l'institution scolaire (Laurent, 2008). La conjonction de ces différents paramètres autorise l'élaboration d'une ingénierie didactique, sous la forme d'un ensemble d'activités à proposer aux élèves, qui fait l'objet d'une mise à l'épreuve dans les conditions ordinaires d'exercice du métier. L'analyse des productions des élèves s'opère en référence aux travaux linguistiques et psycholinguistiques et permet une première évaluation de l'ingénierie proposée. En effet, cette mise à l'épreuve en conditions réelles fournit des éléments susceptibles d'interroger la pertinence et l'opérativité du dispositif par rapport aux difficultés d'apprentissage constatées, la congruence et la compatibilité des activités proposées avec le fonctionnement habituel des pratiques d'enseignement (Garcia-Debanc, 1991 : 17). Mais la validation de la pertinence de cette ingénierie pour les apprentissages visés n'est pas alors suffisante. Elle suppose une mise à l'épreuve dans des classes nombreuses et diverses, d'une part en vue d'observer les éventuels heurts ou contradictions entre les activités ainsi proposées et les pratiques ordinaires des enseignants, d'autre part en vue d'évaluer les effets sur les apprentissages visés à court, moyen et long terme. Dans le cadre de cette contribution, nous ne pourrons prendre en charge que les premières étapes de ce travail, à savoir la conception et la mise à l'épreuve dans une classe d'une ingénierie didactique prenant en compte les acquis de recherches linguistiques et psycholinguistiques.

La construction des séances proposées est d'abord motivée par l'existence d'une question d'enseignement, bien que les verbes de déplacement ne figurent pas explicitement dans les progressions des programmes de l'école primaire $2008^{(3)}$. On peut toutefois considérer que les verbes de déplacement relèvent des termes afférents aux actions et qu'un travail dans ce domaine s'inscrit dans les prescriptions du programme. Un travail explicite sur les verbes de déplacement se justifie par un besoin récurrent en situation d'écriture : la localisation spatiale et la des-

(3) Ceux-ci indiquent que, au CE 2, devront être travaillés les «termes appartenant aux lexiques des repères temporels, de la vie quotidienne et du travail scolaire», au CM1 « les termes afférents aux actions, sensations et jugements» et au CM2 «les termes renvoyant à des notions abstraites (émotions, sentiments, devoirs, droits) ». 
cription de déplacements font partie intrinsèque de la narrativité, au même titre que la dimension temporelle. Les récits, très souvent pratiqués à l'école élémentaire et au collège, supposent des déplacements de personnages, que ce soit pour les genres qui y font directement référence, comme les récits de voyage ou les contes merveilleux initiatiques, mais aussi, plus largement, pour tous les genres de récits, nouvelles policières, récits d'aventures ou récits d'expériences. Le déplacement spatial, notamment sous la forme d'un éloignement de la maison, de la famille figure dans la liste des fonctions constituant la base morphologique des contes merveilleux (Propp, 1928). Ainsi la première fonction est libellée de la sorte : « un des membres de la famille s'éloigne de la maison» (Propp, 1970:36), la fonction XI correspond au cas où « le héros quitte sa maison » (ibid. : 50), la fonction $\mathrm{XV}$ «le héros est transporté, conduit ou amené près du lieu où se trouve l'objet de sa quête (définition : déplacement dans l'espace entre deux royaumes, voyage avec un guide, désigné par G) (ibid. : 63). Six formes (plus ou moins) spécifiques de déplacement sont énoncées :

1. - Le héros vole dans les airs (G1). A cheval (171), sur un oiseau (210), à la manière d'un oiseau (162), dans un bateau volant (138), sur un tapis volant (192), sur le dos d'un géant ou d'un esprit (210), dans la calèche du diable (154), etc. 2. - Il se déplace sur la terre ou l'eau (G2). A cheval, ou sur le dos d'un loup (168). Dans un bateau (247).

3. - On le conduit. Un peloton de fil lui montre le chemin (234).

4. - On lui indique le chemin.

5. - Il utilise des moyens de communication immobiles (G5). Il gravit une échelle (156), découvre une entrée souterraine et l'utilise (141).

6. - Il suit des traces de sang (G6).

Les déplacements sont également mentionnés dans les fonctions XX : « le héros revient (définition : retour), XXI : «le héros est poursuivi» mais aussi X-XI bis «le héros repart, recommence une quête », XV bis « le héros est transporté ou amené près du lieu où se trouve l'objet de sa quête » ou XXIII : « le héros arrive incognito chez lui ou dans une autre contrée » (ibid. : 69,74). Les déplacements spatiaux occupent donc une place importante dans les fonctions de base du conte merveilleux.

Dans un article s'interrogeant sur les démarches pertinentes dans l'étude de la langue pour répondre aux difficultés rédactionnelles des élèves, Masseron (2001) a montré que les notations de déplacements sont quasiment absentes dans des productions narratives d'élèves de $6^{\mathrm{e}}$. Les verbes locatifs les mieux représentés sont des verbes intransitifs de polarité initiale (sortir) ou finale (revenir). En contrepoint, l'analyse d'un extrait de récit d'aventures permet de mesurer la complexité et la diversité des procédés possibles dans un texte littéraire.

La conception de séances d'enseignement est facilitée par la disponibilité de travaux linguistiques sur les verbes de déplacement, présentés notamment par Borillo (1998), Vieu (1991), Guillet-Leclère (1992), Laur (1991). Borillo (1998 : 131) définit les verbes de déplacement comme décrivant « les phases d'un changement spatio-temporel que subit la cible », définie comme « objet à localiser relativement à un lieu, ou dans le cas présent du mouvement, l'entité qui se déplace ou est déplacée » (objet humain ou non humain)» ${ }^{(4)}$ (Laur, 1991:39) par rapport à un repère, appelé « site », « soit que la cible seule effectue le déplacement, soit que le

(4) Définition établie d'après Vandeloise (1987) cité par Borillo (1998) : «l'élément que l'on cherche à localiser $"$. 
déplacement affecte à la fois site et cible ». Certains verbes contraignent le site : on ne peut s'immerger que dans un lieu de nature liquide, $s$ 'embourber que dans de la boue, s'empaler que sur un objet pointu, tel un pal. D'autres verbes, comme transvaser ou empoter contraignent à la fois la cible et le site. Borillo (1998) distingue quatre structures syntactico-sémantiques, selon que l'argument site et cible est sujet ou complément. Elle propose également une classification d'ordre sémantique, tenant à la polarité aspectuelle du verbe. Borillo (1998) distingue ainsi :

- les verbes de polarité initiale ou « verbes initiaux», comme partir, s'enfuir, sortir, s'échapper, s'éloigner, quitter... : « le déplacement exprimé par le verbe prend implicitement le site comme lieu d'origine de la cible».

- les verbes de polarité finale ou "verbes finaux », tels que arriver à, atteindre, entrer dans, regagner... «L'emplacement du site représente la destination vers laquelle la cible se déplace ou est déplacée, et où elle est censée se trouver une fois le déplacement effectué » (Borillo, 1998: 133).

- les verbes de polarité médiane ou « verbes médians » correspondent aux cas dans lesquels « la zone du site représente le lieu parcouru ou traversé par la cible durant son déplacement ». Ces verbes ne constituent pas une catégorie aussi homogène que les précédentes, dans la mesure où, "selon le cas, le site peut représenter 1'endroit où se trouve déjà la cible avant son déplacement et où elle est encore après, ou bien correspondre au parcours ou à une portion du parcours de la cible pendant son déplacement ». Si circuler dans, flâner dans, se déplacer dans correspondent au premier cas de figure, traverser, parcourir, passer par correspondent plutôt au deuxième cas.

Ces divers travaux, ainsi que ceux qui portent sur 1'acquisition du lexique verbal par les enfants (Duvignau, 2005) ont été utilisés pour mettre en place des activités dans une classe de CM2 engagée dans un projet d'écriture d'un épisode supplémentaire de l'album de François Place, Le Roi des Trois Orients (Ed. Rue du Monde). L'hypothèse est qu'un travail de classement des verbes d'un domaine donné permet une meilleure appropriation de ce lexique et l'emploi approprié de verbes variés dans des productions écrites.

Après l'énoncé des principes généraux sous-jacents à un travail lexical au service de la production écrite, nous présenterons le déroulement de ce projet puis nous procéderons à une analyse des classements proposés par les élèves à propos d'un ensemble de verbes de déplacement, présentés en annexe 3.

\section{Enseignement du lexique et production écrite : travail sur la synonymie ou sur la co-hyponymie?}

Les prescriptions scolaires les plus courantes demandent de chercher un synonyme pour éviter une répétition ou de remplacer un verbe support comme mettre ou faire par un terme plus spécifique. Or, le travail d'élaboration des formulations apparaît plus complexe que les injonctions données. En effet, ce qui distingue le rédacteur qui utilise un vocabulaire « fondamental », limité à 2000 à 3000 mots et la personne cultivée qui mobilise un vocabulaire actif de 25.000 à 30.000 mots, part infime des 250.000 entrées du Trésor de la Langue Française Informatisé, c'est bien de choisir avec soin le terme spécifique pertinent dans un contexte donné. On peut se demander quelles ressources peuvent être mises à disposition des rédacteurs pour ouvrir les possibilités de ces choix et quelles activités d'enseignement lexical sont susceptibles de développer chez les élèves non seulement les connaissances lexicales mais aussi le goût et l'exigence du choix des mots. 
Si l'on considère, avec Marina Yaguello, dans Alice au pays du langage, (p. 190), que « les signes de la langue ne se laissent définir que par et relativement à d'autres signes dont ils sont solidaires dans le système autonome de la langue », chaque mot ne prend valeur que par rapport à d'autres mots du système avec lesquels il peut commuter sur un axe paradigmatique. Chacun des mots présente des nuances de sens, que l'on perçoit mieux par contraste avec d'autres mots de sens proche. Le travail de formulation passe parfois par une recherche de synonyme mais il implique aussi très souvent la recherche d'un terme plus spécifique, dans une relation d'hyperonymie / hyponymie. Les isonymes ou co-hyponymes sont des mots partageant une même relation hiérarchique avec un hyperonyme : cèpe, morille, girolle ne sont pas synonymes mais sont tous des co-hyponymes de champignon (Lehmann, Martin-Berthet, 2008 : 92). Les co-hyponymes peuvent entretenir entre eux des relations de synonymie (jovial et guilleret sont des co-hyponymes de gai) ou d'antonymie (acheter et voler sont des antonymes co-hyponymes de se procurer). La recherche du terme spécifique est facilitée à l'écrit par l'absence de contrainte temporelle. Elle implique donc non seulement une activation des relations de synonymie mais aussi de celles d'hyponymie et co-hyponymie.

\subsection{Place du lexique dans les modèles psycholinguistiques du processus rédactionnel}

Les modèles du processus rédactionnel, et plus largement de la production verbale orale ou écrite, élaborés par les psychologues du langage (pour une présentation synthétique, voir Fayol, 1997 et Fayol et alii, 2002) donnent moins d'indications sur les opérations de formulation que sur les opérations de planification. Lorsqu'ils s'efforcent de rendre compte de l'accès lexical dans la production verbale orale ou écrite, le modèle le plus reconnu, celui de Levelt (1989) présente l'accès lexical comme s'effectuant sous la forme d'un module autonome et procédant en deux étapes successives : une sélection lexicale sémantique et syntaxique de lemmas, nœuds de relations conceptuelles interconnectés ou éléments lexicaux abstraits, précède l'encodage phonologique des unités lexicales et leur insertion syntaxique (cité par Fayol, 1997 : 64). La distinction entre «lemma », comme entité abstraite préphonologique et unité lexicale repose sur 1'observation de divers phénomènes, notamment l'analyse des erreurs de production par des participants sains, les lapsus analysés par Garrett (1980), le phénomène du mot sur le bout de la langue, l'analyse des performances de patients cérébrolésés ainsi que des expériences de dénomination en temps réel à partir d'images par des participants normaux. Il est à noter que ces travaux expérimentaux portent sur la dénomination de mots ou la production de phrases et non sur des productions textuelles. Tous ces travaux convergent pour mettre en avant l'activation de nœuds conceptuels préalablement aux formulations des mots eux-mêmes.

\subsection{Que nous disent les ratures des écrivains et des élèves sur le travail lexical dans l'élaboration des formulations? L'apport de la génétique textuelle}

L'examen des ratures des écrivains experts (Fuchs 1982) ou des écoliers (Fabre-Cols, 2002) fournit également des indications intéressantes sur les opérations de lexicalisation. S'intéressant à la paraphrase dans des brouillons de Proust, Fuchs (1982) montre que « la lexicalisation est un processus qui intervient à tous les moments de la production d'un énoncé (aussi bien au niveau des opérations 
énonciatives que prédicatives) et qui peut porter aussi bien sur un élément isolé que sur toute une séquence d'éléments mis en relation » (Fuchs, 1982 : 93).

Dans les brouillons d'écoliers examinés par Fabre-Cols (2002), le remplacement est l'opération la plus fondamentale, dans la mesure où elle représente $45 \%$ de 1'ensemble des modifications sur 1'ensemble du corpus (Fabre-Cols, 2002 : 79). Elle intervient dans toutes les phases de l'écriture, même si une grande part des modifications se joue au niveau infra-lexical.

\subsection{Quelques principes pour l'enseignement du lexique}

L'examen des travaux linguistiques et psycholinguistiques permet de s'accorder sur quelques grands principes pour définir un enseignement du lexique efficace dans la perspective de la production d'écrits :

- Un travail spécifique en langue est nécessaire.

- Il importe de travailler sur des mots fréquents et polysémiques plutôt que sur des mots rares et de mettre en relation le vocabulaire nouveau avec le vocabulaire déjà connu.

- Le vocabulaire n'est intégré que s'il est réutilisé dans plusieurs situations, à 1'oral et à l'écrit, en réception et en production.

- Il importe de veiller à ce que le travail lexical porte sur les noms mais aussi sur les adjectifs et les verbes. Les verbes notamment, du fait de leur fréquence et de la difficulté de leurs constructions syntaxiques, doivent faire l'objet d'une attention particulière.

- Comme les mots ne s'emploient pas isolément et comme de nombreuses erreurs tiennent à la construction syntaxique, il importe de travailler les différentes constructions syntaxiques des verbes ou collocations (Polguère, 2008).

- Le travail sur le lexique est un espace privilégié pour construire des aides à la production écrite. Celles-ci peuvent être construites par la classe à l'issue d'un travail en langue sur le lexique ou être constituées par des dictionnaires d'encodage (Pruvot 2006 : 146), conçus selon une approche onomasiologique.

\subsection{Quelles ressources peuvent apporter certains dictionnaires ?}

Alors que la plupart des dictionnaires qui nous sont familiers relèvent d'une approche sémasiologique, c'est-à-dire visent à expliquer les différentes significations des mots comme signes, un certain nombre de dictionnaires illustrent une approche onomasiologique, allant des idées aux mots qui permettent de les traduire dans la langue. Ces dictionnaires, bien représentés dans le monde anglo-saxon depuis de nombreuses années, connaissent actuellement un grand développement dans l'édition française, dans la mesure où, depuis quelques années, chaque éditeur en publie. Leur ancêtre pour le français est le Thésaurus, publié par Larousse en 1991, qui se définit comme «fondamentalement un outil à transformer les idées en mots et les mots en idées ». Les mots y sont regroupés en termes génériques, auxquels renvoie un index. Ainsi, pour trouver le verbe aller, il faut consulter 1'article 197 MOUVEMENT 18. aller, circuler, migrer 677 ; se promener, voyager 869. Bouger, se déplacer, se mouvoir, se remuer ; fam. avoir la bougeotte, gigoter, 19. accélérer 576 ; se presser ${ }^{(5)}$.

(5) 869 VOYAGE v. 19 Voyager; partir, battre le pays, voir du pays; faire du chemin, faire le 
Dans une perspective d'écriture, sont également très utiles des dictionnaires de synonymes, tel que le Dictionnaire de synonymes, mots de sens voisin et contraires, édité par Quarto Gallimard 2007 et présenté comme 1'outil de référence d'écrivains comme Anne Gavalda. De très nombreux synonymes sont proposés, par ordre alphabétique, pour le verbe aller, sans que les significations de ces différents termes soient spécifiées :

ALLER v. intr s'acheminer, affluer, s'approcher de, avancer, cheminer, cingler vers, circuler, converger, courir, déambuler, se déplacer, se diriger, s'écouler, faire route sur / vers, filer, gagner, galoper, se jeter dans, marcher, mettre le cap sur, se mettre en route, se mouvoir, parcourir, passer par, pérégriner, piquer sur, se porter / poursuivre / pousser / progresser vers, se promener, remonter, se rendre à, suivre, tendre / tirer / tourner ses pas, se transporter sur / vers, traverser, voyager vers

Divers dictionnaires sont également présents sur la toile, comme le dictionnaire des synonymes du CRISCO, présenté par ailleurs dans ce numéro.

Les listes de termes proposés dans ces divers dictionnaires constituent une mémoire externe des ressources disponibles en langue. Toutefois, lorsqu' on écrit, une liste de ces mots ne suffit pas. Pour les utiliser de façon pertinente dans un texte, encore faut-il connaître leurs significations spécifiques, leurs conditions d'emploi ainsi que les constructions syntaxiques dans lesquelles ils entrent (Polguère, 2004). Pour être efficaces, ces listes aide-mémoire nous semblent donc devoir référer à des apprentissages lexicaux effectués antérieurement par les élèves.

\section{Un travail en langue : classification d'une liste de verbes de déplacement. Analyse des classifications proposées par des élèves de CM2}

L'hypothèse qui sous-tend notre travail est de considérer qu'une tâche de catégorisation lexicale de verbes liés par une relation de proximité sémantique implique une meilleure mémorisation de ce lexique par les élèves et la mobilisation pertinente de verbes spécifiques dans une tâche de production écrite. Un ensemble de verbes de déplacement est donc proposé, sélectionnés à partir du logiciel PROX (Gaume, 2003).

Les activités de classification sont pour les élèves l'occasion de préciser la signification des différents verbes, leurs contextes d'emploi, d'en dégager des traits communs de signification, de mettre en évidence des nuances de sens. La confrontation des classifications proposées permettra d'en vérifier la pertinence mais aussi de conduire les élèves à réutiliser ces verbes dans de nouveaux contextes.

\subsection{Une référence : la classification des verbes de déplacement proposée par des travaux linguistiques}

Laur (1991 : 86) observe que les verbes à polarité initiale ou finale « forment incontestablement la classe la plus importante numériquement ». Elle considère que « l'aspect télique exprimé par ces verbes (différence entre deux localisations) semble être le plus significatif de l'expression du déplacement en français ». Elle

tour du monde, pérégriner (vx ou litt.) - S'aérer, changer d'air, se dépayser.

V 21 Se déplacer; se balader (fami), se promener ; ambuler (litt), déambuler, errer, flâner, marcher; cheminer, excursionner, randonner 
observe que « la deuxième catégorie très importante numériquement et qui constitue une liste ouverte est la catégorie des verbes décrivant avant tout une manière de se déplacer ». Elle indique en effet que «la langue crée un nouveau verbe à chaque expérience nouvelle dans le domaine de la locomotion (skier, surfer, varaper, escalader, ramer, patiner...) » parce que « nous attachons beaucoup d'importance aux diverses manières de se déplacer (déplacement lié aux divers moyens de transport ou à différents sports » (ibid.). Nous verrons que les élèves sont très sensibles à cette dimension dans les classifications qu'ils proposent. En revanche, on trouve peu de verbes décrivant une orientation ou une direction du déplacement (avancer ou reculer, s'éloigner, s'approcher) ou décrivant une localisation différente lors des phases temporelles du déplacement (avant, pendant et après) (traverser, transiter, passer par, couper par).

Laur (1991 : 87) montre par ailleurs que les verbes finaux ou initiaux et les verbes médians ne se regroupent pas de la même manière. En effet, à l'intérieur de la liste des verbes téliques, on peut constituer des paires de verbes qui s'opposent seulement par leur polarité initiale ou finale : arriver / partir, entrer / sortir, venir / s'en aller, atterrir / décoller, emménager / déménager, immigrer / émigrer... On trouve également quelques paires dans la catégorie des verbes initiaux et finaux d'orientation : avancer / reculer, s'approcher /s'éloigner, devancer / suivre, précéder/succéder à.. En revanche, cela est impossible pour les verbes à polarité médiane. Ces différences sont si fortes que Vandeloise (1987 : 85) ne considère pas les verbes médians comme des verbes de déplacement mais comme des « verbes de manière de déplacement. Si les verbes marcher, courir, ramper... entraînent un déplacement, il reste de toute façon à l'arrière-plan, la fonction essentielle de ces verbes étant de nous apprendre la manière dont le mouvement s'effectue $»$.

Les verbes de déplacement peuvent être présentés en combinant les deux critères de classification sous la forme de quatre ensembles ou types, comme le propose Borillo (1998) ${ }^{(6)}$ :

- Le type 1 correspond à des verbes de déplacement transitifs, comme quitter, atteindre, longer, cotoyer, traverser, sillonner, qui se construisent selon une structure de type N0 cible Vdyn N1 site ${ }^{(7)}$. Guillet et Leclère (1992) recensent 130 verbes de ce type. Ces verbes peuvent être :

- des verbes de polarité initiale, comme quitter, évacuer, abandonner, déserter

- des verbes de polarité finale, comme atteindre, regagner, rejoindre, réintégrer, percuter

- des verbes médians comme arpenter, sillonner, explorer, gravir, grimper, parcourir, traverser...

- Le type 2 correspond à des verbes transitifs pour lesquels c'est le site et non la cible qui remplit la fonction de sujet, comme dans l'énoncé :

La mer a rejeté une épave de bateau.

On peut grouper dans cette catégorie les verbes rejeter, cracher, avaler, vomir, diffuser, inhaler... Guillet et Leclère ne recensent pas moins de 160 verbes répondant à cette construction.

(6) Voir aussi 1'article de Sénéchal (Willems, 2007), qui reprend les principales classifications des verbes trivalenciels locatifs.

(7) Vdyn : verbe dynamique. 
- La forme canonique de l'expression du déplacement est portée par la troisième catégorie, constituée par des verbes transitifs indirects, selon des constructions du type N0cible Vdyn Prép N1site, comme dans les énoncés suivants :

Paul sort dans le jardin.

Le chat se réfugie sous la table.

Paul court après la balle.

Il existe en français un très grand nombre de verbes se construisant selon ce modèle, qu'ils soient simples ou pronominaux : Laur (1991) en dénombre 500. Parmi ces verbes, les plus nombreux sont des verbes de polarité finale, comme arriver, entrer, se poser, s'échouer, revenir, se réfugier, atterrir, plonger, approcher, se rapprocher. Guillet et Leclère (1992) dénombrent 220 verbes de ce type et plus de 120 verbes de polarité initiale, tels que partir, s'enfuir, émigrer, sortir, s'échapper, s'éloigner... Les verbes médians sont difficiles à dénombrer, dans la mesure où cette catégorie regroupe des verbes indiquant le parcours suivi par la cible, comme les verbes passer par, transiter par, couper par, précédant un complément prépositionnel introduit par par ou à travers mais aussi un très grand nombre de verbes indiquant un déplacement de la cible à l'intérieur d'un même lieu de référence, comme circuler, marcher, rouler, nager, errer, se déplacer, courir, vagabonder, voler. Ce dernier ensemble de verbes est constitué pour l'essentiel de «verbes qui décrivent des modes de déplacement (quelque chose comme se déplacer en Vant) ». (Borillo, 1998)

- Le quatrième type de construction correspond aux verbes comme fourmiller ou grouiller qui ont pour sujet un N0site, comme dans 1'énoncé :

La place grouille de touristes.

Ces verbes sont souvent interprétés comme des verbes de localisation statique.

Pour le travail en classe, nous n'avons retenu que les types 1 et 3 proposés par Borillo (1998) comme étant clairement des verbes de déplacement et correspondant aux constructions les plus fréquentes.

\subsection{Présentation du corpus proposé}

Le corpus de verbes proposés à l'observation des élèves et des adultes est identique : il est constitué d'une liste alphabétique de 37 verbes, choisis parmi les premiers cités par le logiciel PROX (Gaume 2003) par rapport au verbe aller. La liste de ces verbes est reproduite en annexe 2.

Nous avons exclu les sens des verbes non relatifs aux verbes de déplacement, comme lorsque aller signifie convenir.

La diversité des verbes proposés entraîne des classement complexes et multicritèriés. Plusieurs classements sont possibles, certains verbes pouvant figurer dans deux ensembles. Si l'on applique les catégories proposées par Borillo (1998), on peut constater que cet ensemble comporte :

- quatre verbes de polarité initiale,

- trois pronominaux intransitifs : s'échapper, s'élancer, s'enfuir

- et un transitif : quitter

- trois verbes de polarité finale :

- deux intransitifs : arriver, revenir

- un verbe transitif : atteindre 
- et de nombreux verbes médians. Parmi ceux-ci, on peut distinguer ceux qui décrivent

- un franchissement d'un espace, tous transitifs (franchir, traverser, parcourir)

- une modalité de déplacement rapide (courir, se précipiter, galoper) ou lente (flâner, traîner)

- vers le haut (escalader, grimper) ou vers le bas (trébucher ?),

- dans un élément particulier, eau ou air : naviguer, nager, ramer, voler

- d'une manière particulière : glisser, sauter, sautiller, galoper

- avec un instrument particulier : ramer, rouler

- un déplacement relatif par rapport à une autre cible : devancer, suivre, distancer, poursuivre, se rapprocher. Ces verbes sont particulièrement nombreux dans l'échantillon proposé.

- un déplacement humain de manière métaphorique mais qui s'appliquent initialement à l'animal : galoper ou ramper.

- un déplacement à l'intérieur d'un espace sans but : errer, flâner, roder, traîner

Restent alors trois verbes génériques : aller, marcher, venir.

On peut considérer que, sauf injonction particulière dans la consigne, des classifications fondées sur des critères syntaxiques n'apparaîtront pas de façon spontanée dans les propositions de regroupements des élèves ou des enseignants. Avec des élèves de fin d'école primaire, on peut considérer qu'il faut plus particulièrement insister sur 1'emploi des verbes transitifs : atteindre, devancer, distancer, escalader, franchir, parcourir, poursuivre, quitter, suivre, traverser. Des activités de productions de phrases permettant leur réemploi peuvent être proposées.

Le but de l'activité n'est pas que les élèves parviennent à un classement type comme celui que nous avons proposé. Le matériau est suffisamment divers pour qu'on puisse observer quels traits de regroupement apparaissent saillants pour les élèves.

\subsection{Les classifications des élèves de CM2}

Organisés en une dizaine de groupes de 2 ou 3, les élèves de CM2 ont proposé des classifications assez diverses (notre annexe 2).

Un seul groupe (le groupe A) a procédé au classement des 37 verbes. Il propose de les regrouper en quatre ensembles :

- destination précise : arriver, atteindre, poursuivre, se précipiter, quitter, se rapprocher, revenir, suivre, venir

- traîner à un endroit : s'échapper, errer, s'enfuir, rôder, traîner, flâner, trébucher

- aller en l'air : sauter, voler, sautiller, s'élancer

- avancer (lent ou rapide) : aller, courir, devancer, distancer, escalader, franchir, galoper, glisser, grimper, marcher, nager, naviguer, parcourir, ramper, ramer, rouler, traverser.

On comprend bien les rubriques, qui mettent en jeu, pour les deux premières d'entre elles, des éléments liés à la polarité aspectuelle des verbes, pour les deux autres, des traits sémiques ayant trait au mode de déplacement. En revanche, les termes regroupés sous ces rubriques posent question. En effet, dans la première rubrique, appelée par les enfants « destination précise», les élèves placent à la fois des verbes qui relèvent des « verbes à polarité finale ", comme arriver, atteindre mais aussi des verbes autres, comme se précipiter ou suivre, voire des verbes de 
polarité initiale, comme quitter. Il est dès lors difficile de comprendre la logique de ces regroupements.

Les autres groupes d'élèves n'ont retenu qu'un nombre plus ou moins important de verbes, de 12 à 32 , le plus souvent une quinzaine.

Certaines des classifications proposées sont clairement identifiées. Ainsi, le groupe $\mathrm{C}$, après avoir retenu initialement deux catégories («verbes de déplacements normals » / « verbes de déplacements rapides »), classe 19 verbes en 4 catégories :

- sur 1'eau : nager, naviguer, ramer

- en 1'air: sauter, sautiller, voler

- en montant : escalader, grimper, franchir, atteindre

- déplacements rapides : courir, s'élancer, s'enfuir, galoper, poursuivre, suivre, se précipiter, s'échapper, parcourir

Cette classification fait intervenir l'élément dans lequel s'effectue le déplacement (« eau », « air » et terre, non spécifié ici), la direction du mouvement («en montant », mais on pourrait attendre aussi la rubrique antonyme, " en descendant ») et la vitesse. Certains des verbes classés le plus tardivement ne répondent pas aux propriétés de la rubrique. Ainsi franchir, atteindre n'impliquent pas un mouvement ascendant, de même que la signification des verbes suivre ou parcourir n'implique pas la vitesse.

Le groupe $\mathrm{H}$ regroupe 32 verbes en quatre ensembles de taille inégale et justifie son classement de la façon suivante :

- aller, galoper, glisser, marcher, nager, naviguer, quitter, ramer, ramper, rouler, sauter, sautiller, traîner, traverser, venir, voler, courir. (devancer, franchir, distancer sont barrés). Car on sait qu'il se déplace et comment mais on ne sait pas où il va, s'il a un but ou pas.

Sont écrits en plus petit grimper, trébucher, escalader, s'élancer, se précipiter, parcourir, franchir, sans doute ajoutés après la formulation du critère de classification.

- errer, flâner. Parce qu'on (barré et remplacé par il) ne sait pas où on va.

- s'échapper, poursuivre, suivre, s'enfuir. Car il a un but

- arriver, atteindre. Car le personnage parvient à son but.

Il semble qu'intuitivement ce groupe fasse référence à la polarité aspectuelle du verbe, telle que la définit Borillo (1998). En effet, le premier ensemble regroupe pour l'essentiel des verbes à polarité médiane, à l'exception du verbe quitter. De même, le second ensemble de verbes regroupe des verbes de significations proches correspondant à un sous-ensemble des verbes à polarité médiane, même si l'inventaire n'est pas exhaustif. De la même manière, le quatrième ensemble correspond très clairement à des verbes à polarité finale et le critère est clairement énoncé. La classification est moins nette pour le troisième ensemble de verbes, qui semblent correspondre en partie à des verbes de polarité initiale, comme s'échapper et s'enfuir mais cette catégorie comporte également des verbes marquant un déplacement relatif comme poursuivre ou suivre. Le verbe quitter, de polarité initiale, étant regroupé avec des verbes médians dans le premier ensemble, on peut considérer que cette valeur sémantique est plus difficile à percevoir pour ce groupe que les autres polarités, qui sont clairement mises en évidence dans la classification proposée et les justifications données. 
Les classements sémantiques ne sont pas toujours faciles à expliquer. Certains groupes, comme le groupe G, ne formulent pas de justification : « On a trouvé dans le dictionnaire c'est mot là ensemble ». D'autres formulent des classements fondés principalement sur l'identité thématique, dans lesquels le chercheur a peine à voir un véritable classement sémantique. Ainsi, le groupe B a ventilé 25 verbes en deux catégories :

- ce qui signifie « SPORT» : escalader, courir, grimper, nager, naviguer, parcourir, ramer, rouler, sauter, voler, glisser, franchir

- ce qui signifie arriver ou partir ou avancer : arriver, atteindre, franchir, aller, poursuivre, devancer, traverser, se précipiter, s'élancer, revenir, s'échapper, quitter, distancer

Le plus souvent, les classifications proposées font intervenir autant de critères de classification que de rubriques, l'ensemble du classement n'obéissant pas à un principe de classification cohérent. Ainsi le groupe $\mathrm{G}$ a traité 15 des 37 verbes en les regroupant en 4 ensembles :

- Verbe qui exprime un déplacement difficile : parcourir, traverser, franchir, atteindre, escalader, grimper

- S'évader d'un endroit : s'échapper, s'enfuir, se précipiter

- Se déplacer sur ou sous l'eau : nager, ramer, naviguer

- Déplacement simple : aller, courir, marcher

La classification semble distinguer des verbes relativement génériques (aller, courir, marcher), qu'ils qualifient de « déplacement simple», Les verbes de polarité initiale (s'échapper, s'enfuir) sont clairement identifiés mais ils sont groupés avec un verbe qui n'a pas cette propriété (se précipiter) et la rubrique correspondant aux verbes de polarité finale n'est pas formulée. Comme d'autres groupes (groupes E ou I), ce groupe crée un ensemble de verbes correspondant à un « déplacement sur ou sous l'eau » mais d'autres modes de déplacement ne sont pas envisagés.

De même, le groupe I a autant de critères de classification que de rubriques, lorsqu'il dégage quatre ensembles :

- ceux où on ne se presse pas : errer, flâner, rôder, traîner, marcher

- ceux où on se presse : courir, s'enfuir, se précipiter, devancer, distancer, poursuivre, s'échapper

- ceux où on va quelque part (ou arrive) : aller, arriver, se rapprocher, marcher, parcourir, venir, revenir, rouler, suivre, voler

- ceux où on est dans sur 1'eau : nager, naviguer

Une mise en commun collective permet de faire apparaître les principaux critères de regroupement. Les catégories validées au tableau reprennent les regroupements qui reviennent de façon récurrente dans les affiches des élèves. Les verbes sont ainsi regroupés en six ensembles :

- partir d'un endroit / aller à un endroit

- aller vite / aller lentement

- suivre quelqu'un

- aller avec un moyen de transport

- monter / descendre

- se déplacer avec difficulté 


\section{Un projet d'écriture littéraire impliquant l'utilisation de verbes de déplacement mis en ouvre dans une classe de CM2 et en formation initiale et continue d'enseignants}

\subsection{L'interaction lecture / écriture au service de l'écriture littéraire}

La tâche d'écriture choisie consiste à ajouter un épisode supplémentaire à un récit imaginaire littéraire. Elle n'est pas un simple exercice d'application permettant de mobiliser le lexique acquis au cours d'un travail en langue.

Le premier avantage de cette tâche d'écriture est qu'elle présente un intérêt culturel. L'univers imaginaire de François Place était familier aux élèves de la classe de CM2 avec laquelle nous avons travaillé. Le voyage et l'exploration sont en effet des thématiques récurrentes dans l'œuvre de François Place, que ce soit dans ses premiers ouvrages documentaires, publiés chez Gallimard, pour lesquels François Place est seulement illustrateur, Le livre des voyages (inédit), Le Livre de la découverte du monde (1986), Le livre des conquérants (1987), Le livre des navigateurs, Le Livre des explorateurs et Le livre des Marchands, ou dans ses albums fictionnels, Les derniers géants (1992) et l'Atlas des géographes d'Orbae, dont il est à la fois auteur et illustrateur. Son œuvre « invite à des voyages dans le temps et dans l'espace de l'imaginaire comme du réel » (Delattre, Dupin, 2003). Le périple décrit par François Place dans Le Roi des Trois Orients est un voyage interminable, loin des repères familiers, à la recherche de l'amitié du Roi des Trois Orients et de sa sagesse. Le format de l'album, supérieur aux formats habituels, autorise, sur chaque double page, une grande image qui occupe les deux tiers de la page, riche en détails sous la forme de personnages dessinés à la plume sur des à plat de couleurs diverses. Le vert, le gris, le jaune ou le brun, dominants selon les paysages traversés, essaient de rendre compte de la diversité des atmosphères, de l'avancée des saisons et de l'épaisseur du passage du temps, thématique récurrente dans ce récit. Ces effets sont renforcés par la reprise des illustrations dans une fresque en trois rouleaux, à la manière des premiers livres chinois, jointe aux premières éditions de l'album. Le déplacement de la caravane est collectif, même si se dessinent quelques figures, comme les Ambassadeurs, les émissaires, le joueur de luth ou la jeune maîtresse de sang royal. Seul le cheval Nuée d'orage a un nom propre. Certaines figures supplémentaires, détourées et dessinées à la plume complètent parfois l'image : c'est le cas d'un dessin d'arpenteurs dans l'une des pages que nous analyserons. Le regard du lecteur aime ainsi à se perdre dans les images, avant d'approcher le texte, peu abondant sur chaque double page mais très dense. Les thématiques du pouvoir de la culture, de la multiplicité des langues, de la temporalité et du souvenir sont constamment présentes, relayées par le motif de la trace. L'imitation et le pastiche de l'œuvre pour y greffer un nouveau passage peuvent permettre aux enfants d'être plus attentifs aux thématiques qui y sont développées.

Dans la tâche d'écriture proposée, le décalage par rapport à l'expérience quotidienne des enfants ne peut qu'être féconde pour l'imaginaire, d'autant plus que le récit est riche de thématiques faisant écho à des peurs possibles : 1'éloignement par rapport à sa famille, la peur de l'inconnu, la naissance d'une petite sœur, la mort d'un père, la disparition d'un cheval, le sauvetage d'une noyade, le déguisement, l'amour secret, les secrets... La diversité des personnages de la caravane, représentés ou mentionnés, permet une identification du lecteur. Même si, quand ils écrivent, les enfants ne le savent pas encore, certaines des épreuves qu'ils devront décrire dans la tâche d'écriture correspondent à des moments de l'album. Il sera 
intéressant ensuite de confronter leur écriture au texte et aux illustrations de l'auteur.

Enfin, le texte de l'album fournit un matériau verbal qui peut être repris ou imité : nom des personnages, thématiques, structures de phrases. Nous faisons l'hypothèse que ce matériau verbal, bien loin de brider l'imaginaire, fournit aux élèves un matériau précieux. De plus, le projet d'écriture les conduit à scruter le texte littéraire avec l'œil de l'artisan ou du bricoleur, attentif au mode de construction. L'écriture est ainsi moteur d'une observation plus attentive du texte littéraire. Des tâches d'écriture imitative permettent ainsi de sensibiliser les élèves, dès l'école primaire, au repérage des caractéristiques stylistiques d'un texte.

\subsection{Déroulement du projet}

Le projet s'est déroulé de manière à peu près identique en classe primaire et en formation initiale ou continue de Professeurs des Ecoles. Nous décrirons ici le travail mis en œuvre dans la classe de CM2 déjà évoquée.

\subsubsection{Contextualisation du projet}

La première phase permet une contextualisation du projet : les premières pages de l'album sont lues à haute voix par l'enseignant et le livre est mis en réseau avec les autres œuvres de François Place, notamment Les derniers géants, œuvre précédemment travaillée par la classe.

\subsubsection{Un premier recensement de verbes de déplacement : activer le vocabulaire passif disponible}

Les élèves sont ensuite invités individuellement à recenser tous les verbes de déplacement qu'ils connaissent et à rédiger une phrase comportant chacun de ces verbes. L'activité dure une quinzaine de minutes.

Ce moment est destiné à faire émerger en vocabulaire actif le lexique du domaine déjà disponible. L'emploi dans des phrases permet de vérifier que les enfants connaissent les constructions des verbes et qu'ils les emploient de façon appropriée. Il est en effet très important que le vocabulaire nouveau acquis puisse être mis en relation avec le vocabulaire déjà connu, même si celui-ci est peu souvent utilisé.

Une première mise en commun orale permet d'établir un inventaire des verbes de déplacement de la classe. Voici la liste des verbes proposés :

planer - glisser - reculer - gambader - virevolter - courir - aller - traverser

- bondir - se déplacer - patiner - grimper - patauger - galoper - marcher

- accélérer - trotter - nager - franchir - sortir - voler - escalader - sauter

- entrer - avancer - (re)venir - gravir - ramper - naviguer - sautiller - partir

- rouler - trottiner - ralentir - se balader - skier - monter - descendre - surfer

- parcourir - passer - cavaler

On note 42 verbes, de fréquences très différentes, parfois traces de lectures antérieures comme gambader, virevolter ou gravir. Certains d'entre eux, comme se balader ou cavaler relèvent d'un registre familier. Beaucoup de ces verbes renvoient à des modalités de déplacement : courir, planer, patiner, nager, voler, naviguer, skier, surfer, rouler. On peut noter également la présence de verbes pouvant s'utiliser pour rendre compte du déplacement d'animaux et utilisables de façon 
métaphorique pour l'homme : planer, bondir, galoper, trotter, ramper, voler. L'inventaire montre parfois comment le lexique est structuré en réseau : ainsi monter appelle descendre mais cet inventaire n'est pas exhaustif : reculer est mentionné mais pas avancer, accélérer mais pas ralentir. On trouve la présence de quelques verbes transitifs, rares dans les productions écrites, comme traverser, franchir, gravir, escalader. Comme nous 1'avions observé dans un travail antérieur (Duvignau, Garcia-Debanc, 2008), le nombre de verbes recensés à travers un tel inventaire est très largement supérieur au nombre de verbes utilisés par les mêmes élèves dans leurs productions écrites. On peut attribuer ceci au fait que leur attention est alors focalisée sur la recherche des mots du vocabulaire passif au lieu d'être occupée aux diverses opérations du processus rédactionnel.

Au début de chacune des séances suivantes, l'enseignant demande aux élèves de rappeler oralement ces verbes, chacun des élèves devant citer un verbe différent. Ce dispositif est destiné à asseoir la connaissance des termes les plus usuels.

\subsubsection{Une analyse du texte littéraire de référence}

La deuxième séance est consacrée à un relevé des verbes de déplacement utilisés par François Place dans les sept premières pages de son album (annexe 1). Cette analyse du texte littéraire permet de constater la diversité des verbes de déplacement utilisés mais aussi la possibilité de répétitions : «C'est la grande Ambassade. Elle marche depuis des mois, peut-être depuis des années. Elle marche sans arrêt, de saison en saison, de pays en pays ». Elle génère l'atmosphère qui permet une première écriture.

Les verbes relevés par la classe sont les suivants :

traverser - monter - passer - précéder - se mettre en chemin - s'écouler - chevaucher - venir - aller - marcher - parcourir - avancer - se retourner - suivre (poursuivre) - dérouter - planer - faire un détour - parvenir - franchir - partir - revenir - s'arrêter - arriver

Certains de ces verbes, notamment précéder et s'arrêter donnent lieu à un débat. On peut d'ailleurs discuter la notation ainsi effectuée. Ainsi, dans la deuxième phrase, monter est utilisé dans l'énoncé : ils montent des chevaux nerveux, faut-il noter monter, comme l'a fait le maître ou monter des chevaux, synonyme de chevaucher employé un peu plus loin dans le texte ? On rencontre là toutes les difficultés liées aux collocations et à la polysémie. Pourquoi n'avoir pas relevé aussi faire longue route ? Planer est ici utilisé de façon métaphorique, de même que s'écouler mais ce dernier verbe décrit le mouvement de la caravane, alors que planent a comme sujets « sa richesse, sa gloire et son renom », intensifiant la thématique du déplacement au-delà de la description de la caravane. Fautil noter les deux verbes sur le même plan?

L'inventaire a été étendu aux noms dérivés de verbes de déplacement, ce qui a permis de revoir des notions de morphologie, notamment la notion de dérivation, et plus particulièrement la nominalisation, travaillée antérieurement :

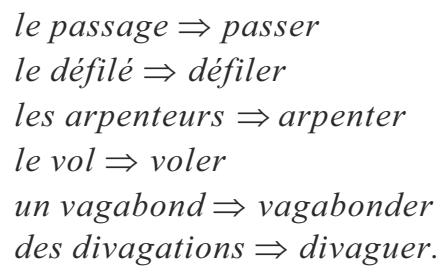


Ce dernier terme a nécessité une recherche dans plusieurs dictionnaires, peu de dictionnaires accessibles aux enfants le donnant dans le sens de déplacement matériel.

Cette mise en relation permet de constater que, le plus souvent, le verbe génère le nom (passer, défiler, arpenter, divaguer) mais que, dans d'autres cas, le verbe est dérivé du nom (voler, vagabonder).

\subsubsection{Une première tâche d'écriture}

La tâche d'écriture proposée, d'une durée de 45 minutes environ, est l'adjonction d'un épisode supplémentaire au voyage de la caravane. La rédaction du texte s'effectue par deux. Le lieu à décrire est obtenu par tirage au sort parmi différents possibles. Les lecteurs devront deviner la situation décrite. Les situations inscrites sur des petits papiers, qui font l'objet d'un tirage au sort, sont les suivantes :

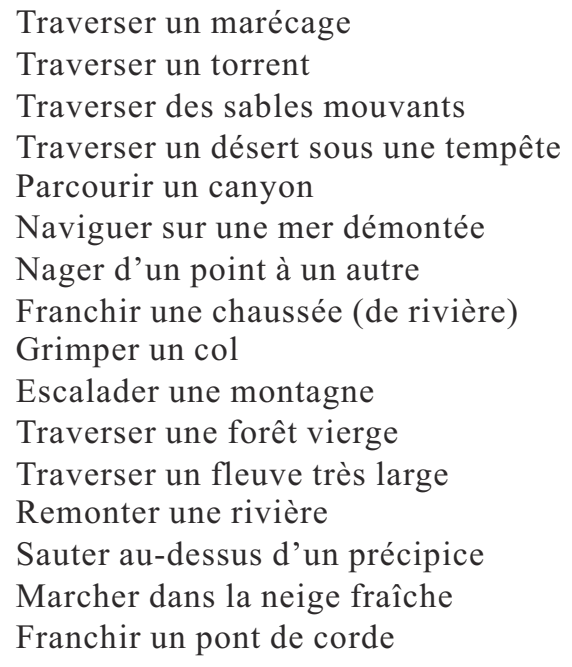

Les rédacteurs peuvent choisir de décrire le déplacement de la caravane ou adopter le point de vue d'un personnage. Ils peuvent s'aider du texte de l'incipit de l'album (annexe 1), des illustrations de l'album ou des trois bandes de volumen vendues avec l'album, fresques des paysages successivement traversés par la caravane.

On peut remarquer que ces situations renvoient à des déplacements difficiles, en milieu hostile, et impliquent l'utilisation de verbes spécifiques et la description des efforts des différents personnages de la caravane pour affronter la situation. Elles nous semblent donc propices à la fois à un travail sur l'imaginaire et à l'emploi de verbes de déplacement spécifiques. Le fait que les lecteurs devront deviner la situation décrite impose de dérouler intégralement un script d'actions, de décrire les déplacements et le milieu sans le nommer directement, ce qui nous semble bien un des enjeux de l'écriture fictionnelle littéraire.

\subsubsection{Un travail systématique en langue sur un échantillon de verbes de déplacement}

Intervient ensuite un travail systématique en langue, sous la forme d'une tâche de classification d'une liste de verbes de déplacement qui a été décrite au point précédent. 


\subsubsection{Une nouvelle écriture}

A l'issue de ce travail est à nouveau proposée la même tâche d'écriture que précédemment. Les élèves n'ont pas sous les yeux le texte qu'ils avaient antérieurement rédigé ${ }^{(8)}$. Nous nous bornerons à citer un seul texte d'élève, représentatif des productions obtenues.

Voici le texte produit par Aïcha, l'orthographe originale a été respectée, les verbes de déplacement sont surlignés en gras.

Vient le moment où une montagne, la plus grande de toutes les grandes montagne se présenta devant la grande Ambassade, la caravane se lança sans perdre espoir à l'assaut de cet énorme bloc rocheux, quand un messager surgit de nulle part :

«Il faut se dépêcher avant que le roi ne désespère! » dit-il.

On se remit à escalader le col, utilisant pioches, cordes, tout se que l'on possedait, le but étant d'atteindre le plus vite possible, la résidence du Roi des Trois Orients.

Par moments, un cavalier glissait de son cheval et commençait à dévaler la pente, roulant de part et d'autre de la caravane mais par miracle, était arrêté par une mule ou un âne, et si ce n'était pas un cavalier, c'était un bœuf qui traînait la marche ou qui s'égarait du groupe et que l'on devait ramener à coups de bâton. Cela faisait plus d'un mois que la caravane avait commencé à gravir la montagne, et les hommes autant que les bêtes s'affaiblissaient, mais reprenaient espoir en penssant qu'ils arriveraient bientôt.

La décente fut un vrai désastre ! Cela glissait tellement que 1'on dut mettre des raquettes, aussi bien aux hommes qu'aux animaux, et ce n'était pas une mince affaire!

Le lecteur pourra constater la diversité des verbes de déplacement employés, ainsi que les effets d'écho par rapport aux formules et au style de François Place.

\section{Conclusion}

Nous souhaitons avoir montré comment, dans un travail de classe, peuvent s'articuler de façon dynamique, pratique des discours, notamment lecture littéraire et production écrite, et analyse de la langue. Le travail présenté a été mis en œuvre dans une classe de CM2 mais la démarche ne serait guère différente au collège. La motivation du travail d'étude de la langue est l'écriture, en amont et en aval d'un travail de langue. Celui-ci repose à la fois sur l'activation du vocabulaire déjà disponible pour les élèves et sur des observations et classifications de mots en discours et en langue : relevé d'occurrences dans des textes littéraires, consultation de différents dictionnaires, classification sémantique d'items appartenant à un même domaine. Le classement des co-hyponymes de verbes génériques, comme ici aller ou même voyager conduit les élèves à dégager le sens spécifique de verbes de sens proche et les incite à utiliser le mot le plus spécifique possible. Le fait que l'étude porte ici sur des verbes compense la désaffection fréquente des exercices des manuels scolaires pour cette catégorie grammaticale.

(8) La situation est proche de celle utilisée par Bucheton (1995) qui demande à des élèves de collège d'écrire plusieurs fois, à plusieurs semaines de distance, le récit d'un même souvenir. Les enjeux de cette écriture ne sont pas identiques, très centrés sur la construction de la personne chez Bucheton, sur le réemploi du lexique enseigné dans le cas présent. 
La taille de cet article ne permet pas d'analyser quantitativement l'emploi des verbes de déplacement dans les diverses productions écrites finales des élèves, de manière à évaluer les effets de la démarche mise en œuvre. Dans une étude antérieure (Duvignau, Garcia-Debanc, 2008), nous avons pu montrer, à partir d'un matériau expérimental mieux contrôlé à propos de verbes co-hyponymes de manger, qu'un travail de classification conduisait les élèves de CM1 à utiliser de façon pertinente un nombre plus important de verbes spécifiques. Dans le cas présent, la mise à l'épreuve de l'ingénierie a fait apparaître le trop grand nombre de verbes, ce qui entraîne, chez les élèves, des classements partiels et dispersés et gêne les controverses au cours des mises en commun. Ces constats ont permis de modifier la liste des verbes soumis à l'activité de classification, la liste figurant dans l'annexe 4 remplaçant la liste initiale pour les expérimentations futures (annexe 3 ).

Un travail du même ordre peut être mis en place pour les verbes de communication ou les verbes et noms de sentiment (Masseron 2008). Du point de vue de la programmation de l'enseignement lexical, les démarches de ce type nous semblent fournir une direction intéressante, sans négliger pour autant les autres dimensions de l'enseignement du lexique, notamment la morphologie et le lexiquegrammaire.

Du point de vue des relations entre didactique et sciences du langage, cette démarche illustre bien, nous semble-t-il, ce que pourrait être une expérimentation didactique faisant appel, selon la formule de Michel Dabène, à une linguistique impliquée. Les modélisations linguistiques proposées à propos des verbes de déplacement (Borillo, 1998, Laur, 1991, Vandeloise, 1987) servent de référence tout au long de l'étude didactique. Non seulement, elles sont sous-jacentes au matériel expérimental soumis aux élèves pour une activité de classification mais elles servent aussi de référence pour l'analyse de leurs réponses et de l'ensemble de leurs productions écrites On peut également souhaiter qu'un examen attentif des classements des élèves, en ce qu'ils témoignent d'intuitions linguistiques, puisse éventuellement permettre d'affiner les modélisations linguistiques en posant de nouveaux problèmes.

Cette démarche a été mise en œuvre, avec quelques aménagements, auprès de Professeurs des Ecoles de deuxième année d'IUFM, en formation professionnelle initiale et au cours de stages de formation continue d'enseignants du premier et du second degrés. Ils ont été placés, eux aussi, en situation de rédiger un épisode supplémentaire au voyage de la caravane après avoir classé un ensemble de verbes de déplacement, celui qui est présenté en annexe 4. Outre les connaissances qu'elle apporte aux enseignants sur les notions sémantiques travaillées, notamment ici les notions de synonymie, co-hyponymie, hyperonymie, une telle démarche contribue également à modifier leurs attitudes quant aux jugements liés à des intuitions sémantiques ou au plaisir de jouer avec les mots de la langue et d'en apprécier le sens précis. Elle leur permet également de prendre connaissance des différents types de dictionnaires et de modifier leur regard sur les outils lexicographiques. L'analyse de leurs productions, que ce soit les textes rédigés ou les classements de verbes qu'ils ont proposés, éclaire les besoins de formation et les compétences professionnelles requises pour conduire efficacement un enseignement du lexique, à savoir une bonne connaissance des relations sémantiques et des outils de référence en langue française mais aussi une représentation pertinente des compétences lexicales des élèves des niveaux considérés. 


\section{Eléments bibliographiques}

BORILlo, A. (1998) : L'espace et son expression en français, Paris, Ophrys.

BRANCA, S. \& GARCIA-DEBANC, C. (2001) : «L'avant-texte à l'école primaire. Quelques jalons de 1850 à nos jours ", dans Fenoglio, I. \& Chanquoy, L. Avant le texte : les traces de l'élaboration textuelle, Langue Française 155, Septembre 2001, 35-50.

Bucheton, D. (1995) : Ecriture. Réécritures. Récits d'adolescents, Bern, Peter Lang.

Butlen, M. et alii (2003) : Les chemins de la littérature au cycle 3, Paris, Scéren, CRDP Créteil, Les chemins de la littérature.

CRINON, J., LEGROS, D. (2002) : «The Semantic Effects of Consulting a Textual DataBase on Rewriting ", Learning and Instruction 12 (6), 605-626.

CRINON, J., PACHET, S. (1995) : «L'aide à l'écriture», Repères 11, Paris, INRP, 33-51.

Delattre, L., DuPIN, A. (2003) : « Exploration des mondes de Morpurgo et Place », in BUTLEN et alii, Les chemins de la littérature au cycle 3, Paris, Scéren, CRDP Créteil, 297-360.

DuVignaU, K. (2003) : «Métaphore verbale et approximation », Revue d'intelligence artificielle RSTI série RIA - Volume 17 - n 5-6 / 2003, Paris, Hermes-Lavoisier, 869-881.

— (2005) : «Pour un apprentissage-enseignement du lexique verbal calqué sur l'acquisition : revisite et apport des "métaphores / erreurs" des enfants de 2-4 ans », in Grossmann, F., Paveau, M.-A., Petit, G. (Eds.) Didactique du lexique : langue, cognition, discours, Grenoble, ELLUG, 37-49.

DUVIGNAU, K., GARCIA-DEBANC, C. (2008) : « Un apprentissage du lexique verbal par proximité sémantique : quand la représentation lexicale facilite la tâche d'écriture ", in Grossmann, F. \& Plane, S. Les apprentissages lexicaux. Lexique et production verbale, Lille, Presses universitaires Septentrion, 17-41.

FABRE-COLS, C. (2002) : Réécrire à l'école et au collège. De l'analyse des brouillons à l'écriture accompagnée, Paris, ESF éditeur.

FAYOL M. (1997) : Des idées au texte. Psychologie cognitive de la production verbale, orale et écrite, Paris, PUF.

FAYOL, M. (éd.) (2002) : Production du langage, Paris, Hermes, Lavoisier.

FUCHS, C. (1982) : «Eléments pour une approche énonciative de la paraphrase dans les brouillons de manuscrits », in Fuchs, C., Gresillon, A., Lebrave, J.-L., Peytard, J., Rey-Debove, J. (éds) La genèse du texte : les modèles linguistiques, Paris, Editions du CNRS, Collection « Textes et manuscrits », 73-102.

GARCIA-DEBANC, C. (2008) : «De la configuration didactique au modèle disciplinaire en acte : trente ans de didactique du français avec Jean-François Halté », in GARCIA-DEBANC, C., MASSERON, C. (eds) Pratiques 137-138, 39-56.

GARRETT, M.F. (1980) : «Levels of processing in sentence production », in Butterworth, B.L (ed) Language Production, Vol. 1 : Speech and talk. New York, Academic Press.

GAUME, B., (2003) : «Analogie et proxémie dans le réseau petits mondes », Revue d'intelligence artificielle RSTI série RIA - Volume 17 - n 5-6 / 2003, Paris, Hermes-Lavoisier, 935-951.

— (2004) : «Balades Aléatoires dans les Petits Mondes Lexicaux », in I3 Information Interaction Intelligence, CEPADUES édition : 39-96 (http://www.revuei3.org/)

GRossmann, F. \& PLANE, S. (2008) : Les apprentissages lexicaux. Lexique et production verbale, Lille, Presses universitaires Septentrion.

GuILLLET, A. (1990) : Les verbes transitifs locatifs, Genève-Paris, Droz. 
GUILlLET, A., LECLERE, C. (1992): La structure des phrases simples en français. Genève, Droz.

Kihlstedt (2005) : In Grossmann, F., Paveau, M.-A., Petit, G. (Eds.) Didactique du lexique : langue, cognition, discours. Grenoble : ELLUG, 85-105.

LAPARRA, M. (2005) : "Capacités langagières en production non fictionnelle », in MASSERON, C. (éd.) Observations de la langue, Pratiques 125-126, Metz, Juin 2005, 139-156.

LAUR, D. (1991) : Sémantique du déplacement et de la localisation en français : une étude des verbes, des prépositions et de leurs relations dans la phrase simple, Thèse sous la direction d'Andrée Borillo, Université Toulouse-Le Mirail.

LAURENT, J.-P. (2008) : «Le savoir enseignant», in DOLZ, J., PLANE, S. Formation des enseignants et enseignement de la lecture et de l'écriture. Recherches sur les pratiques. Actes du symposium de Sherbrooke (Québec). Colloque international REF 9 et 10 octobre 2007, Diptyque 13, Namur, Presses Universitaires de Namur, 223-231.

Lehmann, A., Martin-Berthet, F. (2008) : Introduction à la lexicologie. Sémantique et morphologie, Paris, Armand Colin, Lettres Sup.

LEVELT, W.J.M. (1989) : Speaking : from intention to articulation, Cambridge, MA : MIT Press.

MARIN, B., LEgros, D. (2008) : Psycholinguistique cognitive. Lecture, compréhension et production de texte, Bruxelles, De Boeck.

MASSERON, C. (2001) : "Note critique sur les exercices de grammaire », in GARCIADEBANC, C. (éd), Les textes de consignes, 111-112, Décembre 2001, 209-236. - (2008) : «Pour une topique de la peur : aspects psychologiques, sémiotiques, linguistiques », in Grossmann, F. \& Plane, S. Les apprentissages lexicaux. Lexique et production verbale, Lille, Presses universitaires Septentrion, 161-190.

MorturEUX, M.-F. (1997, 2004, 2006) : La lexicologie entre langue et discours, Paris, Armand Colin.

NOYAU, C. (2003) : «Le lexique verbal dans des restitutions orales de récits en français L2 : verbes de base, flexibilité sémantique, granularité », in Grossmann, F., Paveau, M.-A., Petit, G. (Eds.) Didactique du lexique : langue, cognition, discours. Grenoble, ELLUG : 65-84.

- (2005) : « Processus cognitif de la construction du lexique verbal dans l'acquisition (L1 et L2) », in Revue d'intelligence artificielle RSTI série RIA - Volume $17-\mathrm{n}^{\circ}$ 5-6 / 2003, Paris, Hermes-Lavoisier, 799-812.

PARIOLlaUd, F. (2008) : Verbes : questions de sémantique, Rennes, Presses Universitaires de Rennes.

POLGUERE, A. (2004) : «La paraphrase comme outil pédagogique de modélisation des liens lexicaux », Calaque, E., David, J. (éds) : Didactique du lexique. Contextes, démarches supports, Bruxelles, De Boeck, 115-125.

Propp, V. (1928; 1965, 1970) : Morphologie du conte, Paris, Points Seuil.

PRUVOT, J. (2006) : Les dictionnaires français outils d'une langue et d'une culture, Paris, Ophrys.

REMI-GIRAUD S. (1986) : Le taste-mots dans les arbres. Etude systématique du lexique français, Lyon, CRDP de Lyon.

SENECHAL, M., WiLLEMS, D. (2007) : «Classes verbales et régularités polysémiques : le cas des verbes trivalenciels locatifs », Langue Française 153, Le classement syntactico-sémantique des verbes français, 92-110.

VANDELOISE, C. (1987) : «La préposition à et le principe d'anticipation », Langue Française 76, Paris, Larousse, 77-111. 
VICTORRI, B., VENANT, F. (2007) : « Représentation géométrique de la synonymie », Le Français moderne I.

Yaguello, M. (1992) : Alice au pays du langage, Seuil.

\section{Matériel d'enseignement / ouvrages adressés aux enseignants}

AÏM, P., MAYET-AlBAGnAC, G. (2008) : L'essence des mots. Une pédagogie active du vocabulaire au cycle 3, Paris, Hachette Education.

CELliER, M. (dir.) (2008) : Guide pour enseigner le vocabulaire à l'école primaire, Paris, Retz.

Gerard, R., HAMEAU, C., IMBERT, N. (1998) : Des séquences de vocabulaire pour le cycle 3, Paris, Nathan Pédagogie.

\section{Dictionnaires}

BerTaud Du ChazAud (2007): Dictionnaire de synonymes, mots de sens voisin et contraires, Paris, Gallimard, Quarto.

Boussinot, R. (PRUVOT, J. dir) : Dictionnaire Bordas. Synonymes, analogies et antonymes avec en plus un dictionnaire thématique reclassant 15.000 références, Paris, Bordas.

LE FUR, D. (dir.) (2007) : Le Dictionnaire des combinaisons de mots, Les synonymes en contexte, Les Usuels du Robert.

NiOBEZY, G. et alii (1979) : Dictionnaire analogique, Paris, Larousse.

PECHOIN, D. (dir) (1991) : Thésaurus Larousse. Des idées aux mots, des mots aux idées, Paris, Larousse.

PICOCHE, J. (2002) : Dictionnaire du français usuel, Bruxelles, De Boeck-Duculot.

\section{Annexe 1 \\ Incipit de l'album Le Roi des Trois Orients \\ François Place, Editions Rue du monde}

Un jour, des cavaliers traversent un village. Ils montent des chevaux nerveux. Ils portent de beaux vêtements et parlent d'une voix sonore. Ils réclament du fourrage, de l'eau, de la viande et du pain.

Ces cavaliers précèdent un long cortège d'hommes, de montures et de chariots, qui passent dans le grondement de leurs hautes roues. Des ânes et des mulets, chargés pour faire longue route, des porteurs de bannière, des équipages de toutes sortes et, d'un groupe à l'autre, des exclamations et des éclats de voix. C'est comme si une ville se mettait en chemin, un jour de grand marché, et s'écoulait à travers la campagne. Au bruit de son passage, les paysans et les vignerons cessent leur travail à des lieues à la ronde.

Parmi les voyageurs, quelques personnages de haut rang, richement vêtus, portant longue barbe et chevauchant d'un air grave, semblent des princes en pèlerinage. Ils parlent entre eux des langues différentes et sont visiblement habitués à partager la table des rois. Le défilé n'est même pas interrompu par la tombée de la nuit. Au chant du coq, dans le village encore tout étourdi, quand la poussière soulevée par cette multitude est enfin retombée, on s'interpelle d'une porte à l'autre :

- D'où vient cette caravane ? Où va-t-elle?

C'est la Grande Ambassade. Elle marche depuis des mois, peut-être depuis des années. Elle marche sans arrêt, de saison en saison, de pays en pays. Des arpenteurs mesurent les distances parcourues, des horlogers comptent les heures écoulées aux sabliers, mais la Grande Ambassade avance, sans jamais se retourner, et c'est toujours au devant qu'elle envoie ses messagers. 
Les éclaireurs et les guides ont un œil dans le ciel et une main sur la carte. Ils ont beau remplir les coffres d'itinéraire soigneusement tracés, ils suivent tout aussi bien les pas d'un vagabond, les arabesques d'un vol d'étourneaux... La Grande Ambassade est ainsi : elle préfère les divagations du chemin aux lois de la ligne droite. Trois pas dans le sable suffisent à la dérouter.

La Grande Ambassade va rendre hommage au Grand Roi, le Roi des Trois Orients, qui règne tout là-bas, à l' autre bout du monde. Sa richesse, sa gloire et son renom planent sur les contrées les plus inaccessibles. Mais il faut faire tant de détours pour parvenir aux frontières de son lointain royaume que nul ne sait quand on pourra en franchir les portes.

Régulièrement des émissaires partent à sa recherche et disparaissent derrière l'horizon. Quand ils reviennent, ils ont les cheveux blanchis et le visage brûlé par le soleil. Ils posent un genou à terre et sortent de leurs manteaux des messages chiffonnés.

La Grande Ambassade s'arrête. Elle reprend son souffle.

Les Ambassadeurs lissent pensivement leur longue barbe. Ils font venir les interprètes, maîtres en langues inconnues, et les calligraphes, experts en écriture secrètes, qui peinent à s'accorder sur ces mots venus d'ailleurs. Ils se disputent, lèvent les bras, froncent les sourcils : "Le Grand Roi s'impatiente », disent les uns. "Il ne faut peut-être pas arriver trop tôt », répondent les autres. On monte les tentes, on déroule les tapis. On s'endormira à la belle étoile, en écoutant la voix grave des conteurs.

\section{Annexe 2}

\section{Les verbes de déplacement}

Voici des verbes de déplacement classés dans l'ordre alphabétique.

Classez-les selon leur sens.

\begin{tabular}{|l|l|l|l|}
\hline Aller & s'élancer & naviguer & rôder \\
arriver & s'enfuir & parcourir & rouler \\
atteindre & flâner & poursuivre & sauter \\
courir & franchir & se précipiter & sautiller \\
devancer & galoper & quitter & suivre \\
distancer & glisser & ramer & traîner \\
s'échapper & grimper & ramper & traverser \\
errer & marcher & se rapprocher & trébucher \\
escalader & nager & revenir & venir \\
& & & voler \\
\hline
\end{tabular}

\section{Annexe 3 : classement des verbes}

Groupe $\boldsymbol{A}: 37$ verbes classés.

- destination précise : arriver, atteindre, poursuivre, se précipiter, quitter, se rapprocher, revenir, suivre, venir

- traîner à un endroit : s'échapper, errer, s'enfuir, rôder, traîner, flâner, trébucher

- aller en l'air : sauter, voler, sautiller, s'élancer

- avancer (lent ou rapide) : aller, courir, devancer, distancer, escalader, franchir, galoper, glisser, grimper, marcher, nager, naviguer, parcourir, ramper, ramer, rouler, traverser.

Groupe B : 25 verbes classés.

- ce qui signifie «SPORT» : escalader, courir, grimper, nager, naviguer, parcourir, ramer, rouler, sauter, voler, glisser, franchir 
- ce qui signifie arriver ou partir ou avancer : arriver, atteindre, franchir, aller, poursuivre, devancer, traverser, se précipiter, s'élancer, revenir, s'échapper, quitter, distancer

Groupe $\boldsymbol{C}: 19$ verbes classés.

initialement deux catégories : « verbes de déplacements normals » / »verbes de déplacements rapides »)

- sur l'eau : nager, naviguer, ramer

- en l'air : sauter, sautiller, voler

- en montant : escalader, grimper, franchir, atteindre

- déplacements rapides : courir, s'élancer, s'enfuir, galoper, poursuivre, suivre, se précipiter, s'échapper, parcourir

Groupe D : 12 verbes classés. Absence de justification. Trois ensembles :

- aller, errer, marcher, rôder, traîner

- venir, arriver, atteindre

- courir, s'élancer, galoper, se précipiter

Groupe $\boldsymbol{E}: 13$ verbes classés. la liste de verbes regroupés précède la recherche d'un titre pour la rubrique

- sauter, sautiller, escalader, grimper: ce sont les verbes qui montent

- ramer, nager, naviguer: les verbes de déplacement dans l'eau

- quitter, s'enfuir, s'échapper: partir

- ramper, trainer, trébucher: verbes de déplacement au sol

Groupe $\boldsymbol{F}: 15$ verbes classés.

- être en l'air : voler, sauter, sautiller, escalader

- aller quelque part: aller, arriver, atteindre, s'enfuir, galoper, naviguer, parcourir, se rapprocher, suivre, traverser, venir

Groupe $\boldsymbol{G}: 15$ verbes classés.

- Verbe qui exprime un déplacement difficile : parcourir, traverser, franchir, atteindre, escalader, grimper

- S'évader d'un endroit : s'échapper, s'enfuir, se précipiter

- Se déplacer sur ou sous l'eau : nager, ramer, naviguer

- Déplacement simple : aller, courir, marcher

« On a trouvé dans le dictionnaire c'est mot là ensemble ».

Groupe $\boldsymbol{H}: 32$ verbes classés.

- aller, galoper, glisser, marcher, nager, naviguer, quitter, ramer, ramper, rouler, sauter, sautiller, traîner, traverser, venir, voler, courir. (devancer, franchir, distancer sont barrés). Sont écrits en plus petit grimper, trébucher, escalader, s'élancer, se précipiter, parcourir, franchir, sans doute rajoutés après la formulation du critère de classification.

Car on sait qu'il se déplace et comment mais on ne sait pas où il va, s'il a un but ou pas.

- errer, flâner. Parce qu'on (barré et remplacé par il) ne sait pas où on va.

- «Car il a un but». : s'échapper, poursuivre, suivre, s'enfuir.

- arriver, atteindre. Car le personnage parvient à son but.

Groupe I : 24 verbes classés. Quatre ensembles :

- ceux où on ne se presse pas : errer, flâner, rôder, traîner, marcher

- ceux où on se presse : courir, s'enfuir, se précipiter, devancer, distancer, poursuivre,

s'échapper

- ceux où on va quelque part (ou arrive) : aller, arriver, se rapprocher, marcher, parcourir, venir, revenir, rouler, suivre, voler

- ceux où on est dans sur l'eau : nager, naviguer 


\section{Annexe 4}

\section{Les verbes de déplacement}

Voici des verbes de déplacement classés dans 1'ordre alphabétique.

En vous appuyant sur des critères d'ordre syntaxique (les constructions des verbes) et sémantiques (leur signification), regroupez ces verbes en ensembles en justifiant votre classement.

\begin{tabular}{|l|l|l|l|}
\hline arriver & franchir & partir & rejoindre \\
atteindre & gagner & parvenir & rôder \\
bondir & galoper & se précipiter & rouler \\
s'échapper & gambader & se promener & skier \\
errer & nager & quitter & traîner \\
s'enfuir & naviguer & ramer & traverser \\
flâner & parcourir & ramper & \\
\hline
\end{tabular}

\section{questions de communication 152009} Pathologies sociales de la communication

\section{Dossier coordonné et présenté par Pascal Durand}

Christine Servais, Véronique Servais : Le malentendu comme structure de la communication

David Forest : "L'intelligence collective » : anatomie d'un poncif

Laurent Martin : Censure répressive et censure structurale : comment penser la censure dans le processus de communication?

Elise Vandeninden : L'art-thérapie au secours de la communication. Diagnostic d'une médiation manquée

Thomas Heller : Reconnaissance et gouvernement des salariés. Au-delà du mépris

Béatrice Fleury, Jacques Walter : Penser le Genre en sciences de l'information et de la communication et au-delà

Marlène Coulomb-Gully : Les sciences de l'information et de la communication : une discipline Gender Blind?

Marie-Joseph Bertini : Le Gender Turn, ardente obligation des sciences de

l'information et de la communication

Béatrice Damian-Gaillard, Cégolène Frisque, Eugénie Saitta : Le journalisme au prisme du genre : une problématique féconde

Catherine Gravet : Les historiens des lettres belges sont-ils aveugles au genre?

Françoise Thébaud : Propos d'une historienne des femmes et du genre

Marie-Ėve Thérenty : Pour une histoire genrée des médias

PRIX AU NUMÉRO 20 euros (frais de port de 3,25 euros en sus)

ABONNEMENT ( 1 an, 2 numéros) 32 euros (frais de port de 6,50 euros en sus)

Revue publiée avec le concours du Centre de recherche sur les médiations (Université Paul Verlaine-Metz) et le soutien du Conseil Régional de Lorraine et du Centre National du Livre et du CNRS

Presses universitaires de Nancy•pun@univ-nancy2.fr 